\title{
Nuevo enfoque de la estomatología forense, en base al estudio de mordedura humana en pacientes con lesiones por violencia familiar $y$ lesiones comunes en las localidades del Bajo Piura \\ Forensic Stomatology New Approach, based on the studies of the Human Bite in Patients with Domestic Violence Injuries and Common Injuries along Bajo Piura
}

Giancarlo Jesús Rodríguez Velarde*

Jorge Conde Leurtua** http://dx.doi.org/10.21503/lex.v15i19.1378

* Médico cirujano egresado de la Universidad Nacional de Piura. Magíster en Derecho, con mención, especialidad en Criminalística y Ciencias Forenses por la Universidad Nacional de Piura. Magíster en Salud Pública, especialidad con mención en Salud Familiar y Comunitaria, por la Universidad Nacional de Piura. Doctorando en Medicina por la Unidad de Posgrado de la Universidad Nacional de Piura. Docente en Odontología Legal y Forense por la Universidad Alas Peruanas, filial Piura. E-mail: gijerove2015@gmail.com

** Cirujano dentista. Maestrando en Salud Pública por la Universidad Nacional de Piura. E-mail: jc10415@hotmail.com

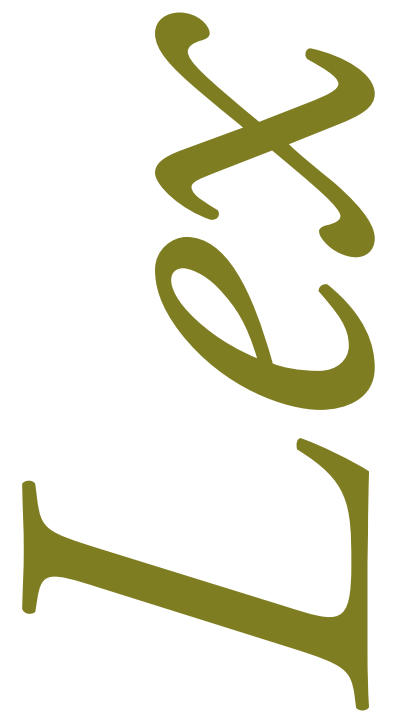




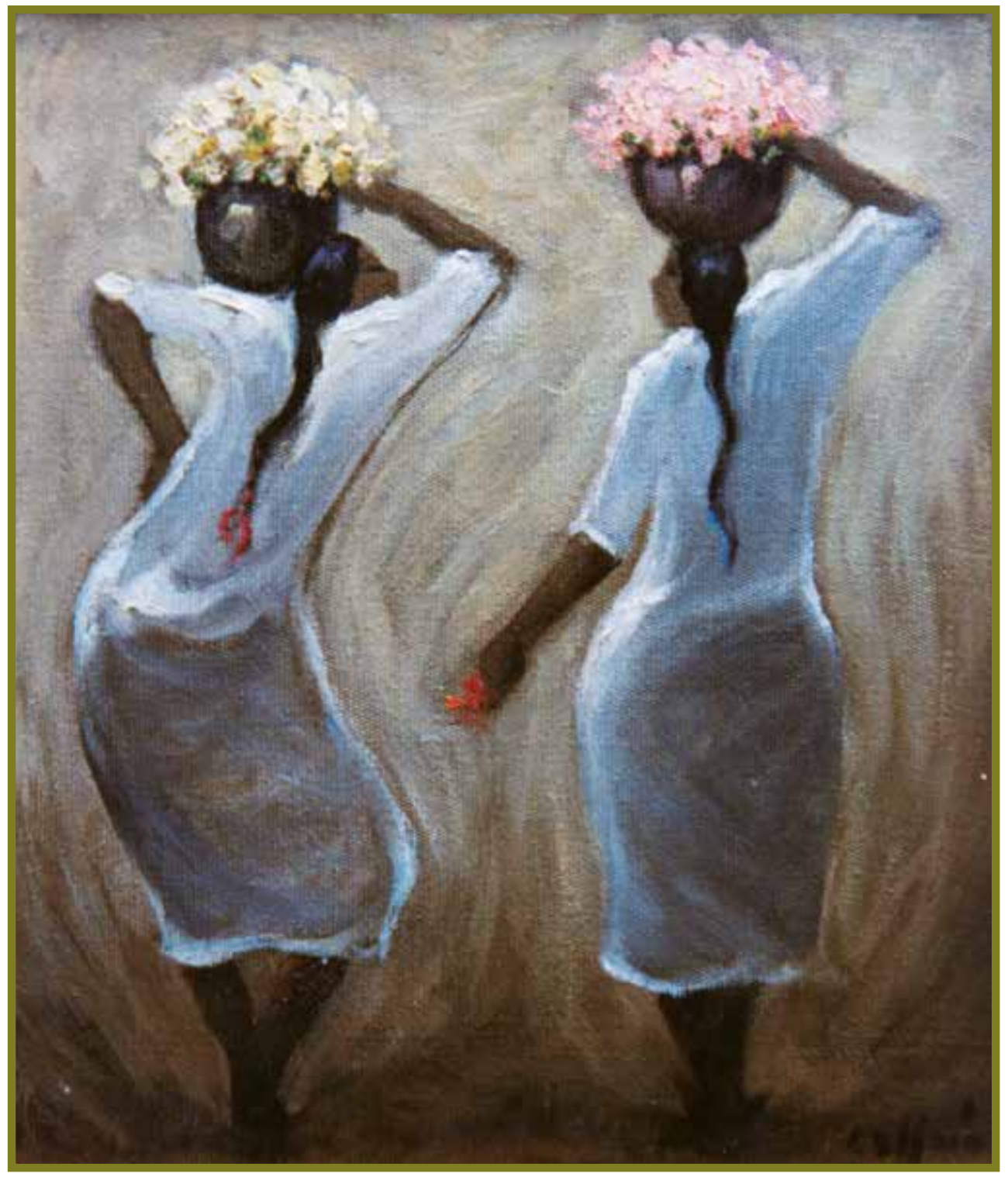

Floristas. Óscar Allaín 


\section{RESUMEN}

Con este trabajo se busca encontrar la relación de mordedura humana en pacientes con lesiones por violencia familiar y lesiones comunes en las localidades del Bajo Piura, en la jurisdicción de la División-Médico Legal I de Catacaos. Se realizó un estudio retrospectivo, exploratorio, transversal y descriptivo de casos y controles del 2009 al 2010. La intervención incluyó la totalidad de la población de acuerdo con los criterios de inclusión y exclusión (2077 casos), y se recolectó la información utilizando una ficha valorada por opinión de expertos, por lo que después se analizaron los datos a través de las pruebas no paramétricas.

Palabras clave: estomatología forense, mordedura humana, violencia familiar, lesiones.

\section{ABSTRACT}

This work intends to find a relation with human bite in patients injured due to family violence and common injuries along Bajo Piura, in the jurisdiction of the Catacaos $\mathrm{N}^{\circ} 1$ Medical-Legal Division. A retrospective, explorative, transversal and descriptive study of cases and controls was carried out from 2009 to 2010. The intervention included the complete population according to the inclusion and exclusion criteria (2077 cases), and the information was collected using a form evaluated by expert opinion, so afterwards the data was analyzed through non parametric tests.

Key words: forensic stomatology, human bite, domestic violence, injuries. 


\section{INTRODUCCIÓN}

Las mordeduras humanas son una parte importante dentro de la odontología legal y forense, ${ }^{1,2}$ y las mordeduras pueden considerarse como marcas figuradas o huellas dejadas por los dientes de humanos ${ }^{3}$ sobre un sustrato que es la piel de sujetos vivos, ${ }^{4}$ que se usan como mecanismo de defensa y/o ataque dentro de la violencia familiar y otros tipos de violencia. ${ }^{5}$

Las mordeduras humanas son lesiones complejas ${ }^{6}$ cuyo análisis incluye el examen cuidadoso y completo de la herida, ${ }^{7}$ para compararla con los rasgos y características de la dentadura del agresor, lo cual es una base de identificación fehaciente a través del método científico, ${ }^{8}$ y fundamental para identificar al agresor en la teoría del caso, a nivel de las fiscalías. En ese sentido, las investigaciones de Barrio y $\mathrm{Col}^{9}$ muestran que "La aplicación de lo que denominamos el método del borde incisal (de identificación de mordedura) para el estudio de las distintas proyecciones fotográficas de marcas de dientes hechas en diferentes zonas anatómicas nos muestran unos resultados con una variación mínima entre los valores de control y el problema Se aprecian diferencias significativas sólo en el caso de los incisivos laterales", por ello "se resalta el papel del odontólogo en la detección del maltrato en la consulta clínica". ${ }^{10}$

C. Girbert y C. E. Villanueva, Medicina legal y toxicología, séptima edición (España: Masson, 2010).

2 P. M. A. Duque, A. G. Velosa y S. S. Carreño, Guía práctica para el odontólogo forense (Bogotá D. C.: Instituto Nacional de Medicina Legal y Ciencias Forenses, 2004).

3 M. M. Hijar, R. L. Flores y R. S. Valdez, Atención médica de lesiones intencionales provocadas por la violencia familiar, (México: Salud Pública, 2003).

4 J. Roca, "Lesiones por mordeduras", http://odonfo.blogspot.pe/2008/07/lesiones-por-mordeduras.html.

5 J. S. Díaz B., J. Carreño R. y J. Quiroz M., Guía médico-legal de valoración integral de lesiones corporales y determinación de deformación de rostro (Perú: Instituto de Medicina Legal y Ciencias Forenses "Leonidas Avendaño Ureta", 2010).

6 H. Moreno, Módulo de medicina legal y servicios públicos de salud (Trujillo: Universidad Nacional de Trujillo, 2009).

7 M. E. Robles Cervantes y E. R. Fernández del Castillo, "Intervención médico-forense y odontología forense en un caso de homicidio", http://webcache.googleusercontent.com/search?q=cache:agmldoxuuhaj:estudiosforenses.mx/articulo. php $\% 3$ fid $\% 3 \mathrm{~d} 4+\& \mathrm{~cd}=3 \& \mathrm{chl}=\mathrm{es} \& \mathrm{ct}=\mathrm{clnk} \& \mathrm{gl}=$ pe.

8 C. A. Guzmán, Manual de criminalística, primera edición (Argentina: La Rocca, 1997).

9 J. E. Muñoz Barrio, J. Gómez García, J. L. Calvo Guirao y A. Luna Maldonado, "Determinación de un eje hipotético en registros incompletos. Su utilidad para la individualización de las marcas de mordeduras humanas", Revista Española de Medicina Legal 37, No 2 (2011).

10 D. M. Estupiñán Torres y G. A. Fontanilla Duque, "Papel del odontólogo en la detección y prevención del maltrato infantil", Univ. Odontol 22, N50 (2002). 
Por otro lado, según el estudio ${ }^{11}$ "es factible utilizar las huellas de mordedura como método de identificación para niños de 6 a 12 años, en caso se presente agresión entre menores o identificando al agresor del menor ya que dejo una huella de mordedura como acto de defensa."

Y en contraste con la realidad, se ha percibido que existe cierta tendencia a distribuirse por la violencia física en la jurisdicción de la DML Catacaos. Por otro lado, la violencia de género y en especial la producida en el ámbito familiar ha recibido un tratamiento prioritario por parte del Estado peruano en los últimos años; sin embargo, aún persisten ciertas situaciones que ameritan que se tomen medidas concretas en esta materia. Según el INEI ${ }^{12}$ (Instituto Nacional de Estadística e Informática), a través de la "Encuesta Demográfica y de Salud Familiar ENDES 2000", la violencia familiar afecta a seis de cada diez mujeres en nuestro país. El $41 \%$ de las mujeres alguna vez ha sido empujada, golpeada o agredida físicamente por su esposo o compañero. Por otro lado, Seldes y $\mathrm{Col}^{13}$ refieren que dentro del maltrato infantil "los elementos utilizados con mayor frecuencia fueron: mano abierta y cinto, puño, palo, lazo, puntapié, cable, quemaduras por fuego (...) mordeduras humanas". En tal sentido, en la presente investigación se determinó la frecuencia de las lesiones tipo mordedura humana en el grupo familiar y fuera de ella dentro del campo de la investigación forense.

Córdova y Vega ${ }^{14}$ realizaron una investigación con el propósito de determinar la prevalencia de la violencia contra la mujer gestante, encontrando que en la zona rural la violencia encontrada se estima en $38,37 \%$ y en la zona urbana fue de $26,48 \%$, encontrándose que el tipo de maltrato más frecuente es la combinación de agresión física más la agresión psicológica en un (43,61\%), existiendo asociación entre violencia y el lugar de procedencia, tiempo de unión y necesidades básicas. Un 26,32 \% tuvo como antecedente violencia dentro de su familia, y el 17,29\% presentó maltrato durante la infancia.

Vélez Londoño y Botero Muñoz $\mathrm{CP},{ }^{15}$ en un trabajo colombiano, encuentran que las mordeduras humanas ocupan un tercer lugar entre las mordeduras por mamíferos $(3,6-23$

11 A. M. Valdivia Flores, "Identificación a través de huellas de mordeduras de niños de 6 a 12 años en el centro de salud Francisco Bolognesi de Cayma, (tesis, Arequipa, 2012), https://tesis.ucsm.edu.pe/repositorio/bitstream/handle/UCS$\mathrm{M} / 4031 / 64.2360$. O.pdf?sequence $=1$ \&isAllowed $=y$

12 Instituto Nacional de Estadística e Informática, A través de la Encuesta Demográfica y de Salud Familiar ENDES (Lima: INEI, 2000).

13 J. J. Seldes, V. Ziperovich, A. Viota, y F. Leiva, "Maltrato infantil: experiencia de un abordaje interdisciplinario", Archivos Argentinos de Pediatría 6, № 106 (2008), http://www.scielo.org.ar/scielo.php?script=sci_arttext\&pi$\mathrm{d}=$ S0325-00752008000600005\&lng=es\&tlng=es

14 J. R. Córdova L. y J. Vega M., Prevalencia y factores asociados de la violencia contra la mujer gestante en la zona rural y urbana, en la provincia de Jaén, setiembre-diciembre 2006 (Lambayeque: Facultad de Medicina Humana de la Universidad Nacional Pedro Ruiz Gallo, 2007).

15 J. D. Vélez Londoño y C. P. Botero, "Acerca de un caso de mordedura humana”, http://www.ciencialili.org/casos-de-interes/acerca-de-un-caso-de-mordedura-humana 
$\%$ de todas las mordeduras) y se asocian con una tasa de complicación más alta que la de las mordeduras por animales.

Sweet, ${ }^{16}$ en un trabajo español, presenta unos lineamientos para el reconocimiento de lesiones por mordeduras, como indicios forenses junto con una perspectiva general del procedimiento recomendado para el análisis y valoración correcta de nuevos casos por el equipo forense.

\section{MATERIALES Y MÉTODOS}

Se realizó un estudio retrospectivo, exploratorio, transversal, y descriptivo, de casos y controles del 2009 al 2010. La intervención incluyo la totalidad de la población de acuerdo con los criterios de inclusión y exclusión, (2077 casos), y se recolecto la información utilizando una ficha valorada por opinión de expertos, por lo que después se analizó los datos a través de la pruebas no paramétricas. La presente investigación es de casos y controles, ${ }^{17}$ y para el recabo de los datos se confeccionó una ficha de recolección donde se detalla el patrón de mordedura humana y otras lesiones no asociados al patrón de mordida. Así mismo se incluyó la existencia de compromiso estético y funcional, ${ }^{18}$ las lesiones que predominan en ambos grupos, su extensión y la data referida de la violencia, contrastada con la data calculada en base al patrón de cambio de color de las lesiones. Para realizar el presente estudio, se contó con el permiso del jefe de potencial humano del Ministerio Público y de la Escuela del Ministerio Público, por lo que se procedió a la revisión de los certificados médicos legales a través del software DICEMEL (software de medicina legal) con datos registrados desde 2009 al 2010, y se procesó la información utilizando el programa Statistical Package for the Social Sciences (SPSS) versión 21, ${ }^{19}$ aplicando como tamizaje la prueba de homogeneidad de Kolmogorov-Smirnov modificado por Lilliefors ${ }^{20}(\mathrm{~K}=0,424 ; \mathrm{p}<0,001)$, [Test de Chi cuadrada. Altamente significativo], determinando que las variables no presentan una tendencia a la distribución normal, por lo que se utilizaron pruebas no paramétricas.

\section{RESULTADOS}

Se trabajó con una población total de 2077 casos, presentando al sexo femenino como grupo predominante con 526 casos $(50,87 \%)$ en violencia familiar y 508 casos $(49,13 \%)$ de lesiones comunes [Lesiones comunes producidas fuera del ámbito familiar.] (Xi = 0,328;

16 D. J. Sweet, “Odontología forense. Análisis de marca de los dientes como indicios forenses”, Ciencia Forense. Revista Aragonesa de Medicina Legal, $\mathrm{N}^{\circ} 7$ (2005).

17 Manual CTO, Epidemiología y estadística, quinta edición (España, 2004).

18 H. Moreno, Módulo de medicina legal y servicios públicos de salud (Trujillo: Universidad Nacional de Trujillo, 2009).

19 S. R. Hernández, C. C. Fernández y L. P. Baptista, Metodología de la investigación, tercera edición (México: Editorial Mc Graw Hill, 2003).

20 W. D. Wayne, Bioestadistica. Base para el análisis de las ciencias de la salud, cuarta edición (México: Limusa Wiley, 2007). 
$\mathrm{p}<0,001)$. El patrón de mordedura humana se presentó en 33 casos $(1,6 \%)$, con una forma de presentación predominante del tipo escoriativo y equimótico, encontrando una mayor prevalencia de lesiones por mordedura humana en lesiones comunes (ámbito no familiar) ( $\mathrm{F}$ $=0,356 ; \mathrm{p}>0,05)$. Dentro de las escoriaciones asociados a patrón de mordedura humana se encontró un promedio de área de la piel afectada en $6,92 \mathrm{~cm}^{2}$ IC $(-7,13 ; 20,90)$, y el de las equimosis, en $4,14 \mathrm{~cm}^{2}$ IC $(-8,73 ; 17,03)$.

TABLA 1. SEXO DEL PACIENTE CON TIPO DE VIOLENCIA

\begin{tabular}{|l|l|c|c|c|}
\cline { 3 - 5 } \multicolumn{2}{c|}{} & \multicolumn{3}{c|}{ TIPO DE VIOLENCIA } \\
\cline { 3 - 5 } \multicolumn{2}{c|}{} & $\begin{array}{c}\text { VIOLENCIA } \\
\text { FAMILIAR }\end{array}$ & $\begin{array}{c}\text { LESIONES } \\
\text { COMUNES }\end{array}$ & TOTAL \\
\hline \multirow{2}{*}{$\begin{array}{l}\text { SEXO DEL } \\
\text { PACIENTE }\end{array}$} & MASCULINO & 138 & 905 & 1043 \\
\cline { 2 - 5 } & FEMENINO & 526 & 508 & 1034 \\
\hline \multicolumn{2}{|c|}{ TOTAL } & $\mathbf{6 6 4}$ & $\mathbf{1 4 1 3}$ & $\mathbf{2 0 7 7}$ \\
\hline
\end{tabular}

GRÁFICO 1. DISTRIBUCIÓN DE LA EDAD DE LOS PACIENTES AGREDIDOS

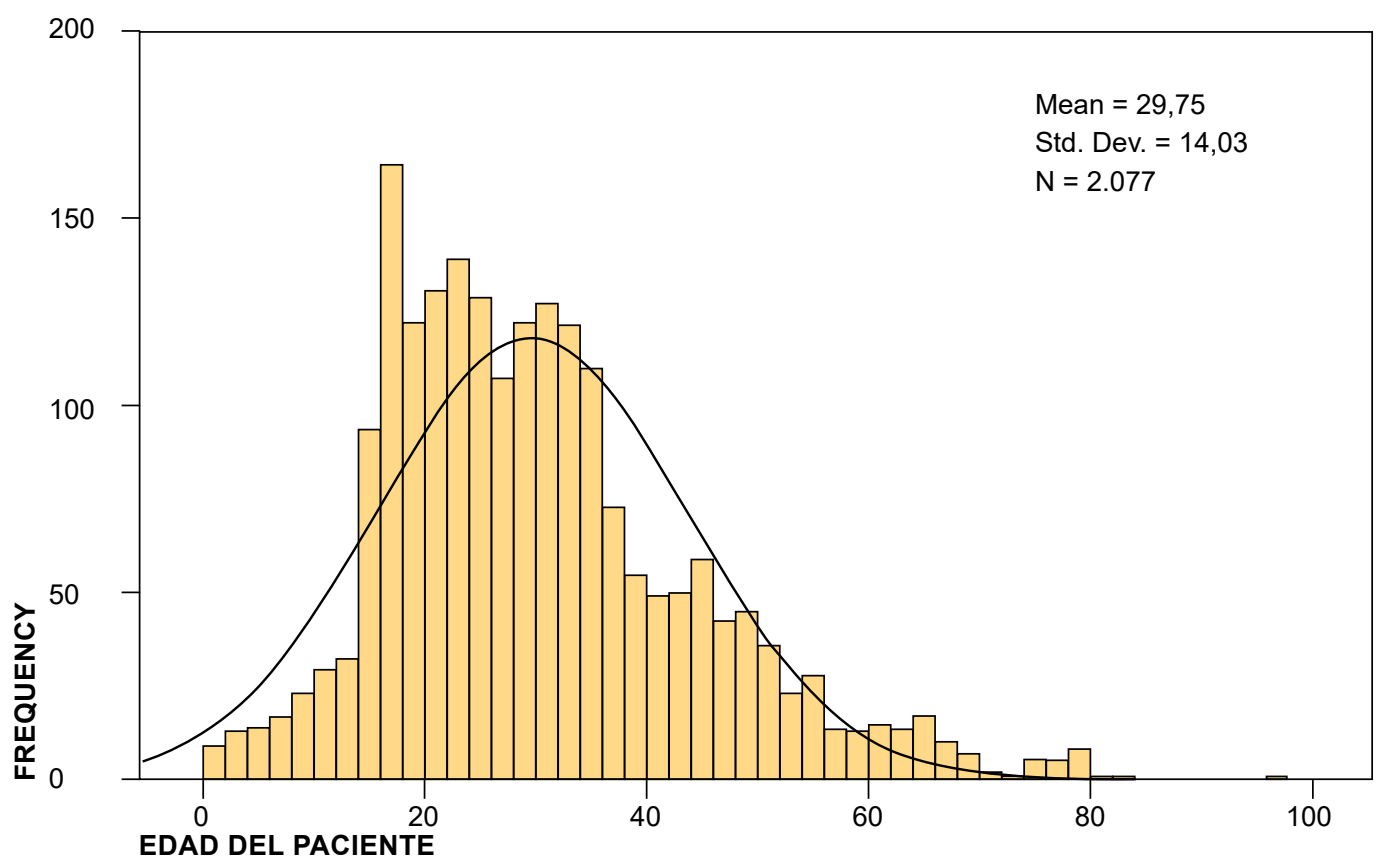


El patrón de mordedura humana se encontró en su mayor localización a nivel frontal, auricular, braquial anterior, dorso y dedos de la mano; y los desprendimientos traumáticos se presentan a nivel de $0,3 \%$ de la población afectada, evidenciándose con más frecuencia las marcas correspondientes a incisivos centrales, laterales y caninos en un $1,4 \%$ y $1 \%$ respectivamente.

En lo que refiere a la localidad de las denuncias, encontramos que los pacientes provienen en su mayor parte de la localidad de Catacaos, representando un 61,7\%, seguida por las zonas rurales de La Arena con 20,5 \%, La Unión con 12,3 \% y por último La Legua con 5,5 $\%$. Encontramos que las variables tipo de violencia y patrón de mordedura humana no están relacionados $\left(\mathrm{Xi}_{2}=0,029 ; \mathrm{p}>0,05\right)$.

GRÁFICO 2. DISTRIBUCIÓN POR LOCALIDAD DE DENUNCIA DE LOS PACIENTES AGREDIDOS

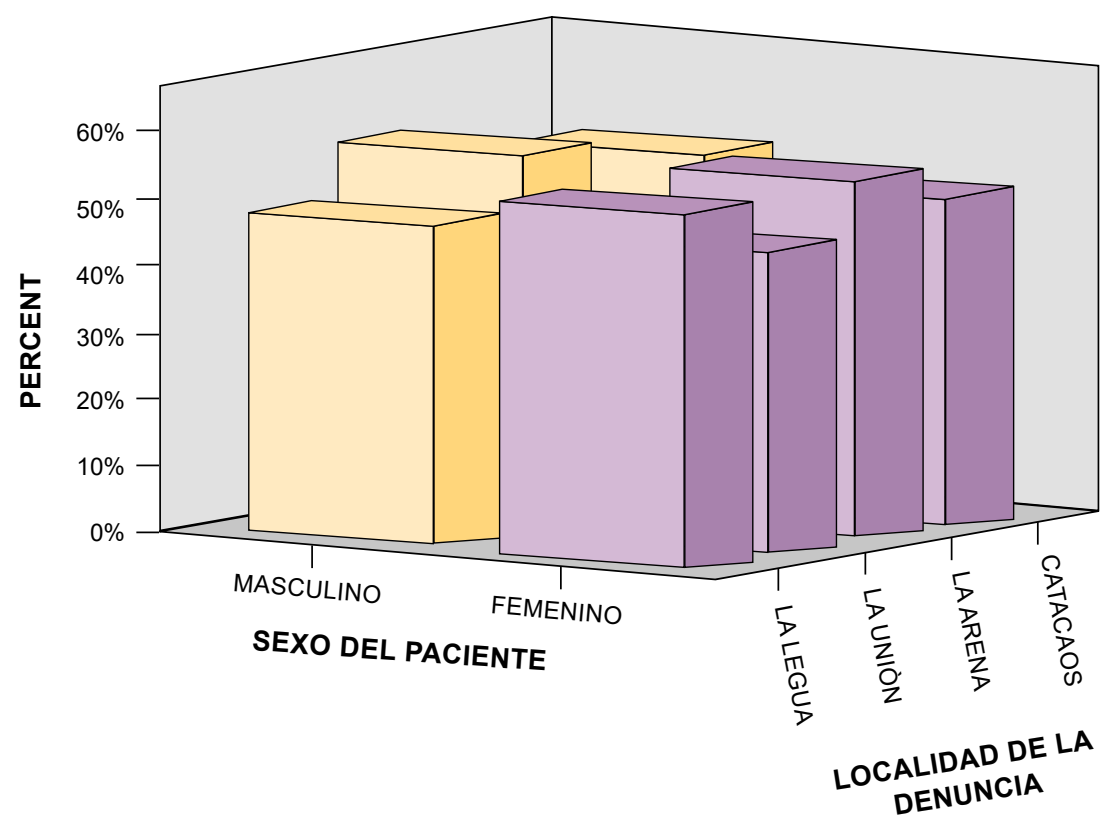

El riesgo estimado para encontrar lesiones por mordedura humana en pacientes que sufran violencia familiar es más bajo $[\mathrm{OR}=0,939$; IC $(0,4-1,9)]$ que el de lesiones comunes, es decir, es un factor protector que representa 1,07 menos riesgo de sufrir lesiones por mordedura en relación a la violencia general. Así mismo, el riesgo estimado o probabilidad de encontrar compromiso funcional temporal en pacientes que sufrieron lesiones por mordedura humana es de 1,159, y siendo más bajo para el compromiso funcional permanente con 0,399 de riesgo (factor protector de 2,51 menos riesgo). Se encontraron cuatro casos de lesiones con 
mordedura humana que presentan compromiso estético en el rostro, un caso de huella indeleble, dos casos de deformación moderada y un caso de deformación severa $\left(\mathrm{Xi}_{2}=9,163\right)$ $(\mathrm{p}<0,05)$, estableciéndose que el patrón de mordedura humana se asocia estadísticamente con compromiso estético en rostro.

Las lesiones se presentaron en 1688 casos, con un promedio de 3,02 lesiones por paciente IC $(-1,34: 7,4)$. El desprendimiento en zonas corporales se encuentra en 52 pacientes $(2,5 \%$ del total).

Por otro lado, con respecto al compromiso funcional temporal, las lesiones traumáticas afectaron a los órganos de los sentidos, encontrando que la visión estuvo afectada en un $80,9 \%$ de los casos (lesionados en el área cefálica), seguida por el olfato con 12,8 \%, la audición con 4,3\%, y por último el sentido del gusto $2,1 \%$. El compromiso funcional temporal se encuentra en 175 casos, con un $8,4 \%$ del total de la población. El compromiso estético en el rostro lo encontramos en 45 casos, y dentro de sus tipos podemos detallar que $1 \%$ de la población presenta huella indeleble, $0,7 \%$ deformación leve, $0,2 \%$ deformación moderada y $0,2 \%$ deformación severa, de los casos estudiados.

El área comprometida en el cuerpo y extremidades se encuentra comprometida en el $51,28 \%$ de los casos, con un promedio de superficie lesionada de $30,98 \mathrm{~cm}^{2}$ IC $(-105,95-$ 167,90); así mismo, el área que se encuentra comprometida en cara y cuello es un 56,19\% de la población, con un promedio de superficie lesionada de $13,45 \mathrm{~cm}^{2}$ IC al $95 \%(-36,5-$ $63,41)$.

Con respecto a la data referida de los pacientes, se comprueba que existe una igualdad de promedios $^{21}$ [Test de U. Mann-Whitney $(\mathrm{Z}=-1,490)(\mathrm{p}>0,05)$. Test de Kolmogorov-Smirnov para dos muestras independientes $(Z=0,069)(p>0,05)]$ entre el tiempo en que se produjeron las lesiones (según versión del paciente) y la fecha de evaluación para ambos grupos; pero en el cálculo de la data a base de las comparaciones del color de las lesiones, observamos que la cronodata conjunta [derivada del patrón de coloración que aproxima la fecha en que se han producido las lesiones] y el tipo de violencia establecen que los promedios no son similares, tendiendo a confluir con un mayor promedio de horas a nivel del grupo con violencia general, a diferencia del grupo con violencia doméstica [test de Kolmogorov-Smirnov ${ }^{22}$ para dos muestras independientes $(Z=-3,256)(\mathrm{p}<0,001)]$, estableciéndose que los pacientes con violencia familiar presentan una mayor preocupación por acudir a los reconocimientos médicos legales, visitando la División Médico-Legal con mayor prontitud para dejar constancia de la interacción violenta, la cual es básica para la identificación del agresor, y así mismo, de la prontitud con que se den las medidas de protección pertinentes.

21 B. Dawson y R. G. Tropp, Bioestadística médica, cuarta edición (México: Manual Moderno, 2005).

22 W. D. Wayne, Bioestadística. Base para el análisis de las ciencias de la salud... 
Por otro lado, se encontró que no existe asociación entre el área comprometida en cara-cuello y extremidades y cuerpo, con la presencia de lesiones compatibles con mordedura humana [Test de U. Mann-Whitney $(Z=-0,848$; p> 0,05) $(Z=-1,253$; $p>0,05)$ ]

\section{CONCLUSIONES}

- De los 2077 casos estudiados, el sexo masculino se encontró como víctima de violencia familiar en el 13,23\% de casos, y en lesiones comunes 86,77\%; por otro lado, la mujer se asocia con violencia familiar en el 50,87 \% de casos y en lesiones comunes $49,13 \%$. Se obtuvo un promedio de la edad del paciente de 29,75 años, y se distribuyó en 2 categorías: la primera que no presenta patrón de mordedura humana en $98,4 \%$ y la segunda que sí presenta en $1,6 \%$. Se encontraron marcas de incisivos centrales y laterales superiores e inferiores con un porcentaje de 1,4\%; caninos superiores e inferiores de $1 \%$. No existe una variación de datos relacionados con la data referida por los agredidos, y que se estandariza con la calculada por los peritos, distribuyéndose. Se encontró que el tiempo difieren una menor proporción en los casos de violencia familiar.

- Existe un predominio del sexo femenino en la localidad de Catacaos en lo referente a violencia familiar, no encontrando una mayor asociación de mordedura humana en pacientes víctimas de violencia familiar, pero sí se encuentra una asociación entre la mordedura y el compromiso estético, con evidencia de marcas de los dientes incisivos y caninos.

- La investigación verificó que las lesiones traumáticas se distribuyen con tendencia al sexo, asociando a la mujer a violencia familiar, y cuya mayor densidad se da a nivel de la comunidad de Catacaos, presentando un promedio de tres lesiones por persona agredida, y un menor tiempo entre la agresión y la fecha del reconocimiento médico-legal para este grupo, siendo importante para la identificación del agresor.

- Las mordeduras humanas se presentan con mayor frecuencia en lesiones de ámbito no familiar, y se encuentran localizadas en las zonas corpóreas anteriores.

- Se evidencia escaso compromiso funcional derivado de la mordedura humana, pero que puede ser seriamente discapacitante cuando se desarrolla, y facilita la identificación y el nexo causal entre el agredido y el agresor.

\section{RECOMENDACIONES}

- Se debería profundizar más en este tema y otros de la rama de odontología forense, y hacer trabajos prospectivos de una cohorte, con mayor número de casos y con períodos de seguimientos. 
- Se recomienda utilizar la cronodata establecida por las bibliografías utilizadas en esta investigación para noción aproximada del tiempo real de la lesión desde que se produjo la misma.

- Que las instituciones públicas como el MINSA, MINDES, Ministerio Público y PNP deben incentivar y continuar los programas de lucha en contra del maltrato infantil y violencia familiar a nivel regional, para disminuir la alta incidencia vista en el presente trabajo.

\section{REFERENCIAS}

- Córdova L., J. R. y J. Vega M. Prevalencia y factores asociados de la violencia contra la mujer gestante en la zona rural y urbana, en la provincia de Jaén, setiembre-diciembre 2006. Lambayeque: Facultad de Medicina Humana de la Universidad Nacional Pedro Ruiz Gallo, 2007.

- Dawson, B. y R. G. Tropp. Bioestadística médica. Cuarta edición. México: Manual Moderno, 2005.

- Díaz B., J. S.; J. Carreño R. y J. Quiroz M., Guía médico-legal de valoración integral de lesiones corporales y determinación de deformación de rostro (Perú: Instituto de Medicina Legal y Ciencias Forenses "Leonidas Avendaño Ureta”, 2010).

- Duque, P. M. A.; A. G. Velosa y S. S. Carreño. Guía práctica para el odontólogo forense. Bogotá D. C.: Instituto Nacional de Medicina Legal y Ciencias Forenses, 2004.

- Estupiñán Torres, D. M. y G. A. Fontanilla Duque. "Papel del odontólogo en la detección y prevención del maltrato infantil”. Univ. Odontol 22, N50 (2002): 90-97.

- Girbert, C. y C. E. Villanueva. Medicina legal y toxicología. Séptima edición. España: Masson, 2010.

- Guzmán, C. A. Manual de criminalística. Primera edición. Argentina: La Rocca, 1997.

- Hernández, S. R.; C. C. Fernández y L. P. Baptista. Metodología de la investigación. Tercera edición. México: Editorial Mc Graw Hill, 2003.

- Hijar, M. M.; R. L. Flores y R. S. Valdez. Atención médica de lesiones intencionales provocadas por la violencia familiar. México: Salud Pública, 2003. 
- Instituto Nacional de Estadística e Informática. A través de la Encuesta Demográfica y de Salud Familiar ENDES. Lima: INEI, 2000.

- Manual CTO. Epidemiología y estadística. Quinta edición. España, 2004.

- Muñoz Barrio, J. E.; J. Gómez García; J. L. Calvo Guirao y A. Luna Maldonado. "Determinación de un eje hipotético en registros incompletos. Su utilidad para la individualización de las marcas de mordeduras humanas". Revista Española de Medicina Legal 37, ํㅜ 2 (2011): 51-58.

- Moreno, H. Módulo de medicina legal y servicios públicos de salud. Trujillo: Universidad Nacional de Trujillo, 2009.

- Robles Cervantes, M. E. y E. R. Fernández del Castillo. "Intervención médico-forense y odontología forense en un caso de homicidio". http://webcache.googleusercontent. $\mathrm{com} /$ search?q=cache:agmldoxuuhaj:estudiosforenses.mx/articulo.php\%3fid\%3d4+\&c$\mathrm{d}=3 \& \mathrm{hl}=\mathrm{es} \& \mathrm{ct}=\mathrm{clnk} \& \mathrm{gl}=$ pe.

- Roca, J. "Lesiones por mordeduras". http://odonfo.blogspot.pe/2008/07/lesiones-por-mordeduras.html.

- Seldes, J. J.; V. Ziperovich; A. Viota y F. Leiva. "Maltrato infantil: experiencia de un abordaje interdisciplinario". Archivos Argentinos de Pediatría 6, No 106 (2008): 499-504. http:// www.scielo.org.ar/scielo.php?script=sci_arttext\&pid=S0325-00752008000600005\&ln$\mathrm{g}=\mathrm{es} \& \mathrm{t} \operatorname{lng}=\mathrm{es}$

- Sweet, D. J. "Odontología forense. Análisis de marca de los dientes como indicios forenses”. Ciencia Forense. Revista Aragonesa de Medicina Legal, Nº 7 (2005).

- Valdivia Flores, A. M. "Identificación a través de huellas de mordeduras de niños de 6 a 12 años en el centro de salud Francisco Bolognesi de Cayma. Tesis, Arequipa, 2012. https://tesis.ucsm.edu.pe/repositorio/bitstream/handle/UCSM/4031/64.2360.O.pdf?sequence $=1$ \&isAllowed $=\mathrm{y}$

- Vélez Londoño, J. D. y C. P. Botero. "Acerca de un caso de mordedura humana”. http:// www.ciencialili.org/casos-de-interes/acerca-de-un-caso-de-mordedura-humana

- Wayne, W. D. Bioestadística. Base para el análisis de las ciencias de la salud. Cuarta edición. México: Limusa Wiley, 2007. 